\title{
Vector algebra and molecular symmetry: a tribute to Professor Josiah Willard Gibbs
}

\author{
Gyula Tasi · László Nagy-Gyevi • \\ Roland Tóbiás · Tamás S. Tasi
}

Received: 27 April 2013 / Accepted: 6 June 2013 / Published online: 19 June 2013

(C) Springer Science+Business Media New York 2013

\begin{abstract}
Vector algebra, as developed by Josiah Willard Gibbs, is much simpler than matrix or tensor algebra, therefore, it is more suitable to introduce the students of chemistry into the wonderful world of molecular symmetry. A program based on elementary vector algebra has been written to determine all symmetry elements and symmetry operations of rigid molecular structures. The program also contains data for 57 point groups common in chemistry. Therefore, it automatically supplies the particular point group to which the structure belongs. Since the locations of the nuclei related to the symmetry elements are also revealed by the program, even the detailed notation of the framework group of the molecular structure can be deduced. The program can be a great help in determining the symmetries of the normal modes of vibration, too.
\end{abstract}

Keywords Molecular symmetry · Symmetry elements and operations · Point group · Framework group · Vector algebra · FORTRAN Language

\section{Introduction}

Symmetry is an important property of molecular shapes [12]. A great number of papers have dealt with the symmetry aspects of molecules in the chemical education literature $[1,2,5,6,10,14-17,20,21]$. For the description of the static symmetry of rigid molecules, the point groups [4] as well as the framework groups [18] are used in chemical education. It is worth noting that framework groups contain more information on molecular shapes than point groups [12].

G. Tasi $(\varangle) \cdot$ L. Nagy-Gyevi · R. Tóbiás · T. S. Tasi

Department of Applied and Environmental Chemistry, University of Szeged,

Rerrich B. tér 1, Szeged 6720 , Hungary

e-mail: tasi@chem.u-szeged.hu 
By static symmetry of a molecule, we mean the symmetry of its equilibrium geometry [7] in ground electronic state. The geometry of a molecule can be specified, for example, via the Cartesian coordinates of its nuclei. These coordinates, however, can also be viewed as a set of position vectors in the common 3-D Cartesian space.

For a symmetric molecular structure, there exist symmetry elements, geometrical entities (points, lines and planes), and symmetry operations, geometrical transformations (inversion, rotations (proper rotations), reflections and rotoreflections (improper rotations)) which move the nuclear framework of the molecule into a position indistinguishable from the original one. It is worth noting that the symmetry operations and not the symmetry elements form the various point groups as defined by Schönflies [4].

In general, matrix algebra is used to determine the main symmetry elements and symmetry operations of a nuclear arrangement. Then, with the help of a simple flowchart $[5,16,6]$, it is a relatively easy task to pick the particular point group to which the molecule belongs [19]. The main point of this procedure is the computation of the molecular inertial tensor which is a 3 by 3 symmetric matrix with real numbers as elements. The authors of a recent paper [9] follow the same strategy even to determine all the symmetry elements. However, vector algebra is much simpler than matrix or tensor algebra, it is therefore more suitable to introduce the students of chemistry into the wonderful world of molecular symmetry. It was Josiah Willard Gibbs (1839-1903) who created the modern vector algebra and vector analysis between 1879 and 1884 [8]. In his works all the properties of the scalar and vector products are fully developed.

It can occur that for larger molecules with peculiar symmetries we might not be able to recognize some important symmetry elements. Furthermore, if we want to build up the particular framework group to which our molecular structure belongs, we must know all the symmetry elements and the precise locations of the nuclei related to them. The same is true when we want to learn the symmetries of the normal modes of vibration. To compute the characters of the symmetry operations in the reducible representation generated by the normal coordinates, we need to know the nuclei which are unmoved by the symmetry operations. In the aforementioned cases, a simple flowchart does not meet our needs.

In the geometry of 3-D space there are points, lines and planes [11]. All these geometrical entities can be represented by vectors in vector algebra: a point as a position vector $\left(\overrightarrow{\mathbf{P}}_{0}\right)$, a line as a position vector with a direction vector $\left(\overrightarrow{\mathbf{P}}_{0}\right.$ and $\left.\overrightarrow{\mathbf{d}}\right)$, and a plane as a position vector with a normal vector $\left(\overrightarrow{\mathbf{P}}_{0}\right.$ and $\left.\overrightarrow{\mathbf{n}}\right)$. Geometrical transformations (inversion, reflection and rotation) simply manipulate the geometrical objects. Since the centre of mass (COM) of a molecule remains fixed in space under all symmetry operations, it is a common point of all axes and planes of symmetry. Accordingly, the COM can be used to define every symmetry element.

A program based on elementary vector algebra has been developed to determine all symmetry elements and symmetry operations of nuclear arrangements. It also contains data for 57 point groups common in chemistry. Therefore, it automatically supplies the particular point group to which the structure belongs. Since the locations of the nuclei related to the symmetry elements are also revealed by the program, even the detailed notation of the framework group of the given molecular structure can be deduced. The program can be a great help in determining the symmetries of the normal modes of vibration. The language of the program is standard FORTRAN 77 
with some extensions accepted even by the popular GNU compiler. The program can be easily extended with further capabilities. The source code can be obtained free of charge from the corresponding author upon request.

\section{A brief outline of vector algebra from the viewpoint of molecular symmetry}

The nuclei of a molecule are points scattered in the 3-D Cartesian space. They can also be viewed as a set of position vectors. Besides addition and subtraction of vectors, there are only two operations in vector algebra which we need in the study of molecular symmetry: scalar (dot, inner) product and vector (cross, outer) product of two vectors.

Let $\overrightarrow{\mathbf{P}}_{1}=\left(x_{1}, y_{1}, z_{1}\right)$ and $\overrightarrow{\mathbf{P}}_{2}=\left(x_{2}, y_{2}, z_{2}\right)$ be two position vectors in the 3-D Cartesian space with lengths $\left\|\overrightarrow{\mathbf{P}}_{1}\right\|$ and $\left\|\overrightarrow{\mathbf{P}}_{2}\right\|$, respectively, and let $\alpha(0 \leq \alpha \leq \pi)$ be the angle between them. Then their scalar product is defined as follows:

$$
\overrightarrow{\mathbf{P}}_{1} \cdot \overrightarrow{\mathbf{P}}_{2}=\left\|\overrightarrow{\mathbf{P}}_{1}\right\|\left\|\overrightarrow{\mathbf{P}}_{2}\right\| \cos (\alpha)=x_{1} x_{2}+y_{1} y_{2}+z_{1} z_{2}
$$

The vector product $\overrightarrow{\mathbf{P}}_{1} \times \overrightarrow{\mathbf{P}}_{2}$ results in a third vector, $\overrightarrow{\mathbf{P}}_{3}=\left(x_{3}, y_{3}, z_{3}\right)$, with

$$
\left\|\overrightarrow{\mathbf{P}}_{3}\right\|=\left\|\overrightarrow{\mathbf{P}}_{1}\right\|\left\|\overrightarrow{\mathbf{P}}_{2}\right\| \sin (\alpha)
$$

and $\overrightarrow{\mathbf{P}}_{3}$ is perpendicular to both $\overrightarrow{\mathbf{P}}_{1}$ and $\overrightarrow{\mathbf{P}}_{2}$, and the triple $\left(\overrightarrow{\mathbf{P}}_{1}, \overrightarrow{\mathbf{P}}_{2}, \overrightarrow{\mathbf{P}}_{3}\right)$ obeys the righthand rule. The components of $\overrightarrow{\mathbf{P}}_{3}$ can be obtained from those of $\overrightarrow{\mathbf{P}}_{1}$ and $\overrightarrow{\mathbf{P}}_{2}$ :

$$
\overrightarrow{\mathbf{P}}_{3}=\left(y_{1} z_{2}-z_{1} y_{2}, z_{1} x_{2}-x_{1} z_{2}, x_{1} y_{2}-y_{1} x_{2}\right)
$$

If the length (norm) of a vector is equal to one, it is said to be normalized.

At the beginning of the analysis of the symmetry of a nuclear arrangement, the $\mathrm{COM}$ of the molecule, $\overrightarrow{\mathbf{P}}_{\text {com }}=\left(x_{\text {com }}, y_{\text {com }}, z_{\text {com }}\right)$, is computed:

$$
x_{\text {com }}=\frac{\sum_{i=1}^{N} m_{i} x_{i}}{\sum_{i=1}^{N} m_{i}} ; \quad y_{c o m}=\frac{\sum_{i=1}^{N} m_{i} y_{i}}{\sum_{i=1}^{N} m_{i}} ; \quad z_{c o m}=\frac{\sum_{i=1}^{N} m_{i} z_{i}}{\sum_{i=1}^{N} m_{i}}
$$

where $N$ is the number of the nuclei, $x_{i}, y_{i}$ and $z_{i}$ are the Cartesian coordinates of nucleus $i$, and $m_{i}$ is the mass of nucleus $i$ considering the most stable isotope of the atom. Next, the origin of the 3-D Cartesian system is shifted to the COM. This is achieved via the subtraction of $\overrightarrow{\mathbf{P}}_{\text {com }}$ from the position vectors of the nuclei.

In our real world, symmetry is never perfect, and the geometry of a molecule is more or less distorted, i.e., it deviates from the perfect ideal one. To measure this distortion, we shall use a distortion parameter, an error limit, within which our statements are valid. For a particular point group or framework group, smaller distortion parameter means more precise molecular geometry. In what follows, the application of such a distortion parameter is tacitly implied in our investigations and statements.

It is easy to check the existence of the centre of symmetry (inversion centre, $i$ ): we should invert the coordinates of the nuclei, and then compare the new configuration 
to the original one. If they coincide, the molecule has inversion centre. If the position vector of one of the nuclei is null (zero) vector, i.e., it has length of zero, that nucleus is then located in the COM.

If all nuclei lie on the same line, the molecule should be linear. In vector algebra this means that the absolute value of the scalar product of any two position vectors of the nuclei divided by the lengths of them is equal to one:

$$
\left|\frac{\overrightarrow{\mathbf{P}}_{i} \cdot \overrightarrow{\mathbf{P}}_{j}}{\left\|\overrightarrow{\mathbf{P}}_{i}\right\|\left\|\overrightarrow{\mathbf{P}}_{j}\right\|}\right|=1
$$

Another possibility is to compute the vector product $\overrightarrow{\mathbf{P}}_{i} \times \overrightarrow{\mathbf{P}}_{j}$, and check whether the result is null vector or not. If it is null vector, the two vectors should be collinear. Of the linear point groups $\mathrm{C}_{\infty \mathrm{v}}$ and $\mathrm{D}_{\infty \mathrm{h}}$ only the latter one has inversion centre.

Another important property of molecular structures is planarity. If all nuclei lie in the same plane, the molecule must be planar. Two, not collinear, position vectors $i$ and $j$ define a plane with normal vector $\overrightarrow{\mathbf{n}}=\overrightarrow{\mathbf{P}}_{i} \times \overrightarrow{\mathbf{P}}_{j}$. If the following equation holds for each nucleus $k$ :

$$
\overrightarrow{\mathbf{n}} \cdot\left(\overrightarrow{\mathbf{P}}_{k}-\overrightarrow{\mathbf{P}}_{c o m}\right)=0
$$

our molecule should be planar: the position vector of each nucleus lies in the molecular plane.

If the molecule is planar and it has inversion centre, it must also have a proper rotational axis $C_{n}$ perpendicular to the plane and passing through the inversion centre. The normal vector $\overrightarrow{\mathbf{n}}$ of the plane can be considered to be the unit direction vector of the rotational axis $(\overrightarrow{\mathbf{d}}=\overrightarrow{\mathbf{n}})$. Now we have to find the smallest angle $\phi$ by which rotating all position vectors of the nuclei in space about the axis represented by the unit vector $\overrightarrow{\mathbf{d}}$, the new configuration is indistinguishable from the original one. The largest $n$ for the given rotational axis is then simply equal to $2 \pi / \phi$.

Let us consider the general case: rotation of the position vector $\overrightarrow{\mathbf{P}}_{k}$ of nucleus $k$ in space by angle $\phi$ about the axis passing through the origin and represented by the unit vector $\overrightarrow{\mathbf{d}}$ as its direction. The new position $\overrightarrow{\mathbf{P}}_{k}^{\prime}$ of $\overrightarrow{\mathbf{P}}_{k}$ can be easily derived:

$$
\overrightarrow{\mathbf{P}}_{k}^{\prime}=\overrightarrow{\mathbf{P}}_{k}+\sin (\phi)\left(\overrightarrow{\mathbf{d}} \times \overrightarrow{\mathbf{P}}_{k}\right)+(1-\cos (\phi))\left(\overrightarrow{\mathbf{d}} \times\left(\overrightarrow{\mathbf{d}} \times \overrightarrow{\mathbf{P}}_{k}\right)\right)
$$

By systematic variation of $n(=2,3, \ldots)$ we can determine every proper rotational axis and rotational operation related to the given rotational axis.

Our final goal is to determine every symmetry element for a given nuclear arrangement. To achieve this goal, we should partition the set of the nuclei into classes, i.e., special disjoint subsets, based on their atomic numbers. The union of such classes is the whole set of the nuclei. Of course, atomic mass numbers must also be considered if we want to deal with isotopomers.

Now we are in a position to determine all planes of symmetry. For possible planes of symmetry, every class of the nuclei is investigated. In a given class we consider 
every $\left(\overrightarrow{\mathbf{P}}_{i}, \overrightarrow{\mathbf{P}}_{j}\right)$ pair of the position vectors of the nuclei. Then planes defined by $\overrightarrow{\mathbf{P}}_{0}$ and $\overrightarrow{\mathbf{n}}$ are created in the following way:

$$
\begin{aligned}
\overrightarrow{\mathbf{P}}_{0} & =\frac{\overrightarrow{\mathbf{P}}_{i}+\overrightarrow{\mathbf{P}}_{j}}{2} \\
\overrightarrow{\mathbf{n}} & =\frac{\overrightarrow{\mathbf{P}}_{j}-\overrightarrow{\mathbf{P}}_{0}}{\left\|\overrightarrow{\mathbf{P}}_{j}-\overrightarrow{\mathbf{P}}_{0}\right\|}
\end{aligned}
$$

If the plane contains the COM, reflections of all the position vectors of the nuclei are performed about it. If the new configuration is indistinguishable from the initial one, a new plane of symmetry is found. For every pair of the nuclei, two further planes with normal vectors $\overrightarrow{\mathbf{n}}_{1}=\overrightarrow{\mathbf{P}}_{i} \times \overrightarrow{\mathbf{P}}_{j}$ and $\overrightarrow{\mathbf{n}}_{2}=\overrightarrow{\mathbf{n}} \times \overrightarrow{\mathbf{n}}_{1}$, respectively, are also checked, if they are not null vectors, for possible planes of symmetry.

Let us consider now the general reflection operation about the plane defined by $\overrightarrow{\mathbf{P}}_{0}=\left(x_{0}, y_{0}, z_{0}\right)$ and $\overrightarrow{\mathbf{n}}=(a, b, c)$. Let $\overrightarrow{\mathbf{P}}_{k}=\left(x_{k}, y_{k}, z_{k}\right)$ be the original position vector of nucleus $k$. By performing the reflection operation, the new $\overrightarrow{\mathbf{P}}_{k}^{\prime}=\left(x_{k}^{\prime}, y_{k}^{\prime}, z_{k}^{\prime}\right)$ position of $\overrightarrow{\mathbf{P}}_{k}$ can be easily obtained:

$$
\begin{aligned}
x_{k}^{\prime} & =x_{k}+2 \lambda a \\
y_{k}^{\prime} & =y_{k}+2 \lambda b \\
z_{k}^{\prime} & =z_{k}+2 \lambda c
\end{aligned}
$$

where

$$
\lambda=\frac{\overrightarrow{\mathbf{n}} \cdot\left(\overrightarrow{\mathbf{P}}_{0}-\overrightarrow{\mathbf{P}}_{k}\right)}{\|\overrightarrow{\mathbf{n}}\|}
$$

The distinct proper rotational axes can be obtained in three steps. First, the position vectors of the nuclei as direction vectors are checked one by one for possible $C_{n}$ axis $(n \geq 2)$. Next, every $\left(\overrightarrow{\mathbf{P}}_{i}, \overrightarrow{\mathbf{P}}_{j}\right)$ pair of the position vectors of the nuclei is investigated for possible $\mathrm{C}_{2}$ axis in each class. The unit direction vector of the rotation axis is simply the normalized form of vector $\left(\overrightarrow{\mathbf{P}}_{i}+\overrightarrow{\mathbf{P}}_{j}\right) / 2$ in this case. In the third step, the remaining $\mathrm{C}_{\mathrm{n}}$ axes $(n \geq 3)$ are to be determined. To carry out this analysis, three nuclei, $\left(\overrightarrow{\mathbf{P}}_{i}, \overrightarrow{\mathbf{P}}_{j}, \overrightarrow{\mathbf{P}}_{k}\right)$, are selected in a given class, and they are considered to be as three adjacent vertices of a regular polygon. By computing -with the help of a dot product- the angle between the vectors $\overrightarrow{\mathbf{P}}_{j}-\overrightarrow{\mathbf{P}}_{i}$ and $\overrightarrow{\mathbf{P}}_{k}-\overrightarrow{\mathbf{P}}_{j}$, we can easily determine the type of the regular polygon, and then check the existence of the corresponding $C_{n}$ $(n \geq 3)$ axis. All unique 3-tuples should be investigated in such a way in each class.

Having the directions of the distinct proper rotational axes, all the proper rotations, the distinct improper rotational axes, and improper rotations can be easily generated by simple rotations. 
If all symmetry elements and symmetry operations are in our hands, we should find the principal axis with possible $\mathrm{C}_{2}$ axes perpendicular to it as well as the horizontal, vertical and dihedral planes of symmetry.

The source code contains data for 57 point groups common in chemistry, therefore, it is easy to identify, without further analysis, the particular point group to which our structure belongs.

Furthermore, for every symmetry element the position vectors of the nuclei which lie in it should be determined. These nuclei are unmoved by the symmetry operations related to the given symmetry element. Special attention should be paid to improper rotational axes and operations. Finally, we will have enough information to deduce the correct notation of the framework group related to our molecular structure.

It is to be seen that everything can be done by simple vector algebra in the study of the symmetry of nuclear arrangements: it is not necessary to use more involved matrix or tensor algebra. The details are to be found in the source code of the program.

\section{An example for illustration: the molecule $\mathrm{N}_{4} \mathrm{~S}_{4}$}

Let us consider an instructive example from a standard book in spectroscopy [3]:

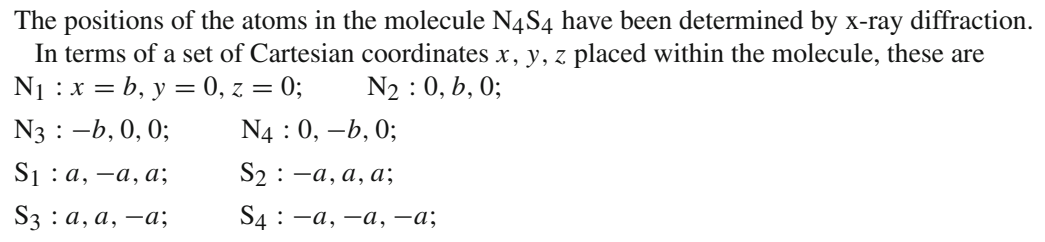

Here the numbers $a$ and $b$ are unrelated parameters of the order of a few angstroms in size.

(a) To what point group does the molecule belong?

(b) What are the symmetries of the normal modes of vibration?

(c) How many different vibrational frequencies does the molecule have?

(d) How many bands should appear in the infrared spectrum as fundamentals?

(e) Assume the parameter $a=3 \AA=1.5 b$. Compute the moments of inertia as well as $A, B$, and $C$. What kind of rotor is the molecule (linear rotor, spherical top, oblate or prolate symmetric top, near oblate or near prolate asymmetric top)?

With 2-D, "paper and pencil", chemistry, it is not always easy to find all symmetry elements and to determine the positions of the nuclei related to them. Let us make an input file ( $\mathrm{n} 4 \mathrm{~s} 4$. inp) from the atomic Cartesian coordinates (see the table below).

It is easy to create an executable program from the source code on every platform: we need only a good fortran compiler. For now we use the GNU fortran compiler under Linux operating system [13]:

>gfortran -o symmetry.exe symmetry.f 


\begin{tabular}{lrrrr}
\hline 7 & 2.0 & 0.0 & 0.0 & ! Atomic number and coordinates \\
7 & 0.0 & 2.0 & 0.0 & \\
7 & -2.0 & 0.0 & 0.0 & \\
7 & 0.0 & -2.0 & 0.0 & \\
16 & 3.0 & -3.0 & 3.0 & \\
16 & -3.0 & 3.0 & 3.0 & \\
16 & 3.0 & 3.0 & -3.0 & \\
16 & -3.0 & -3.0 & -3.0 & \\
\hline
\end{tabular}

To run the executable program, we should enter the following statement:

$>$ symmetry.exe <n4s4.inp > n4s4.out

The output is redirected to the file n4s4 . out. According to the output file, the molecule has three $\mathrm{C}_{2}$ axes (one $\mathrm{C}_{2}$ and two $\mathrm{C}_{2} \perp \mathrm{C}_{2}$ ) and two $\sigma_{\mathrm{d}}$ planes of symmetry. The $\mathrm{C}_{2}$ axis does not contain atoms, however, each $\mathrm{C}_{2}{ }_{2}$ axis and each $\sigma_{\mathrm{d}}$ plane contains two nitrogen and two sulphur atoms, respectively. Two improper rotations can be generated about the $C_{2}$ axis: $S_{4}$ and $S_{4}^{3}$. The results reveal the point group and the framework group of the molecular structure:

$$
\mathrm{D}_{2 \mathrm{~d}}=\left\{\mathrm{E}, \mathrm{C}_{2}, 2 \mathrm{C}_{2}^{\prime}, 2 \sigma_{\mathrm{d}}, \mathrm{S}_{4}, \mathrm{~S}_{4}^{3}\right\}=\left[2 \mathrm{C}_{2}^{\prime}(\mathrm{N} 1 . \mathrm{N} 1), 2 \sigma_{\mathrm{d}}(\mathrm{S} 2)\right]
$$

We can now determine the characters of the symmetry operations in the reducible representation $\Gamma^{3 \mathrm{~N}}$ generated by the normal coordinates [4]:

\begin{tabular}{llllll}
\hline $\mathrm{D}_{2 \mathrm{~d}}$ & $\mathrm{E}$ & $2 \mathrm{~S}_{4}$ & $\mathrm{C}_{2}$ & $2 \mathrm{C}_{2}^{\prime}$ & $2 \sigma_{\mathrm{d}}$ \\
\hline$m$ & 8 & 0 & 0 & 2 & 2 \\
$\Gamma^{3 \mathrm{~N}}$ & 24 & 0 & 0 & -2 & 2 \\
\hline
\end{tabular}

In the second line, $m$ gives the number of unmoved atoms related to the given symmetry operation. With the help of the character table of $\mathrm{D}_{2 \mathrm{~d}}$, we can decompose the reducible representation $\Gamma^{3 \mathrm{~N}}$ into irreducible ones (irreps) [14]:

$$
\Gamma^{3 \mathrm{~N}}=3 \Gamma^{\mathrm{A}_{1}} \oplus 3 \Gamma^{\mathrm{A}_{2}} \oplus 2 \Gamma^{\mathrm{B}_{1}} \oplus 4 \Gamma^{\mathrm{B}_{2}} \oplus 6 \Gamma^{\mathrm{E}}
$$

To get the irreps of the vibrational normal coordinates, $\Gamma^{\mathrm{v}}$, the irreps of the translational $\left(\Gamma^{\mathrm{t}}\right)$ and rotational $\left(\Gamma^{\mathrm{r}}\right)$ normal coordinates should be subtracted from $\Gamma^{3 \mathrm{~N}}$ :

$$
\Gamma^{\mathrm{v}}=\Gamma^{3 \mathrm{~N}}-\Gamma^{\mathrm{t}}-\Gamma^{\mathrm{r}}=3 \Gamma^{\mathrm{A}_{1}} \oplus 2 \Gamma^{\mathrm{A}_{2}} \oplus 2 \Gamma^{\mathrm{B}_{1}} \oplus 3 \Gamma^{\mathrm{B}_{2}} \oplus 4 \Gamma^{\mathrm{E}}
$$

It is to be seen that there are 14 different vibrational frequencies. According to the character table, only the normal modes of vibration with $\mathrm{B}_{2}$ and $\mathrm{E}$ symmetries are infrared active. This means that seven bands should appear in the infrared spectrum 
of the molecule as fundamentals. The last question of the problem is out of the scope of the present paper: it cannot be answered without the analysis of the inertial tensor of the molecule.

The equilibrium molecular structure of allene also belongs to the $\mathrm{D}_{2 \mathrm{~d}}$ point group, however, the locations of the nuclei related to the symmetry elements are quite different in this molecule. This, of course, will be manifested in the notation of the framework group to which the molecular structure belongs. The program correctly reveals the positions of the nuclei related to the symmetry elements. (The analysis of the equilibrium molecular structure of allene is also available in the supplementary material.) One carbon nucleus is in the COM, therefore, this atom is embedded in every symmetry element. Two more carbon nuclei lie in the $\mathrm{C}_{2}$ axis. The remaining proper and improper rotational axes have only one atom. As for the two $\sigma_{\mathrm{d}}$ planes, both contain five atoms from which two are hydrogen. Based on these facts, the notation of the framework group of allene can be easily deduced:

$$
\mathrm{D}_{2 \mathrm{~d}}=\left[\mathrm{O}(\mathrm{C} 1), \mathrm{C}_{2}(\mathrm{C} 1 . \mathrm{C} 1), 2 \sigma_{\mathrm{d}}(\mathrm{H} 2)\right]
$$

It is to be seen that this notation is very different from that of the molecule $\mathrm{N}_{4} \mathrm{~S}_{4}$ even though both molecules belong to the same point group.

\section{Conclusions}

Modern vector algebra created by Josiah Willard Gibbs at the end of nineteenth century can be successfully used to analyse the structure of rigid molecules. The method presented in the paper is much simpler than those based on matrix or tensor algebra. The FORTRAN language has been chosen to implement the procedure. The program determines all symmetry elements and symmetry operations, and supplies the particular point and framework groups to which the structure belongs. It can also be a great help in determining the symmetries of the normal modes of vibration, too.

Acknowledgments The authors thank the TÁMOP project (TÁMOP-4.2.2.A-11/1/KONV-2012-0047) for financial support.

\section{References}

1. J.M. Alvarino, A. Chamorro, J. Chem. Educ. 57, 785 (1980)

2. I. Baraldi, D. Vanossi, J. Chem. Educ. 74, 806 (1997)

3. P.F. Bernath, Spectra of Atoms and Molecules (Oxford University Press, Oxford, 1995)

4. D.M. Bishop, Group Theory and Chemistry (Dover Publications, New York, 1993)

5. R.L. Carter, J. Chem. Educ. 45, 44 (1968)

6. I. Casas, J.J. Pérez, J. Chem. Educ. 69, 83 (1992)

7. J. Demaison, J.E. Boggs, A.G. Császár (eds.), Equilibrium Molecular Structures: From Spectroscopy to Quantum Chemistry (CRC Press) (Taylor and Francis Group, Boca Raton, 2011)

8. J. Johnston, J. Chem. Educ. 5, 507 (1928)

9. R.J. Largent, W.F. Polik, J.R. Schmidt, J. Comput. Chem. 33, 1637 (2012)

10. W.-K. Li, J. Chem. Educ. 70, 485 (1993)

11. J.E. Marsden, A.J. Tromba, Vector Calculus (W. H. Freeman and Company, New York, 2003) 
12. P.G. Mezey, Shape in Chemistry: An Introduction to Molecular Shape and Topology (VCH Publishers, Inc., New York, 1993)

13. J.F. Moore, M.P. McCann, J. Chem. Educ. 80, 219 (2003)

14. B.K. Niece, J. Chem. Educ. 89, 1604-1605 (2012)

15. G.D. Nigam, J. Chem. Educ. 60, 919 (1983)

16. J.H. Noggle, J. Chem. Educ. 53, 190 (1976)

17. M. Orchin, H.H. Jaffé, J. Chem. Educ. 47, 246 (1970)

18. J.A. Pople, J. Am. Chem. Soc. 102, 4615 (1980)

19. G. Tasi, Computational Chemistry (in Hungarian) (JATEPress, Szeged, 2010)

20. E. Vitz, J. Chem. Educ. 79, 896 (2002)

21. M. Zeldin, J. Chem. Educ. 43, 17 (1966) 